\title{
A Statistical Theory of Shape
}

\author{
Rikard Berthilsson \\ Dept of Mathematics, Lund University \\ Box 118, S-221 00 Lund, Sweden \\ rikard@maths. 1th.se
}

\begin{abstract}
In this paper, we will study the statistical theory of shape for ordered finite point configurations, or otherwise stated, the uncertainty of geometric invariants. A general approach for defining shape and finding its density, expressed in the densities for the individual points, is developed. Some examples, that can be computed analytically, are given, including both affine and positive similarity shape. Projective shape and projective invariants are important topics in computer vision and are discussed at the end of the paper.
\end{abstract}

Keywords: Uncertainty, Distribution, Density, Shape, Invariants, Recognition.

\section{Introduction}

In computer vision, it is a common practice to use invariants as a tool for recognising objects in images. This is often done by selecting a fixed number of ordered feature points from each object and then constructing an invariant or, more precisely, an invariant function acting on images of these point configurations. Depending on what type of imaging process that is at hand, e.g. affine or projective camera, one can use affine or projective invariants. If the camera is, for example, projective, then the projective invariant gives the same value when applied to the image of a point configuration independent of what projective camera is used. In order to obtain as much information as possible from the invariant, we would like it to give different values when applied to two point configurations that can not be images of one point configuration. In that case we say that the invariant functions value is the shape of the object.

However, if measurement errors are introduced then there is also uncertainty about the shape. This leads to a problem if we have a data base consisting of a set of shapes representing some objects, that we would like to be able to recognise. If the shape of an unknown point configuration is measured, it is not likely that it is in the data base, due to, for example, the measurements errors. There is then a problem in deciding what element in the data base the measured shape should be identified with. Perhaps it should not be identified with any element at all.

In this paper we present a novel theory for computing the density function for the set of shapes that can occur, when the joint density function of the point 
configurations is known. By knowing this density of shape, the problem above is truly solved. It is then simply a matter of computing confidence sets for the elements in the data base. The shape of a measured unknown point configuration is recognised according to what confidence set/sets it belongs to. If it does not belong to any confidence set, we draw the conclusion that the shape is not part of the data base.

The presentation below is mainly theoretical. It covers both affine and projective shape and there are no assumptions made on the density function of the point configurations. For a more detailed presentation and for all the proofs, we refer the reader to the thesis [2].

The pioneer in the study of the statistical properties of shape is Kendall, c.f. [4], who dealt with the case of positive similarity shape. His work has been further developed in e.g. [5].

In [3] a first order approximation is used to compute the density of the affine shape for planar four point configurations, when the points are normally distributed. A similar method is used in [7].

Densities of projective shape have been studied in [1] and [6], where the density of the cross ratio is computed, for some special cases of densities of the point configurations.

Finally, the density of shape is crucial for a variety of purposes, such as estimating the impact of measurement errors in computer vision algorithms or for finding alignment of stones in archaeological excavations c.f. [4].

\section{Shape of finite ordered point configurations}

In this section, we will define a general notion of shape of finite point configurations. To begin with, we introduce some terminology.

Let $\mathcal{C}_{m}^{n}$ be the set of ordered $m$-point configurations

$$
\mathcal{X}=\left(p_{1}, p_{2}, \ldots, p_{m}\right)
$$

in $\mathbb{R}^{n}$, where $p_{i} \in \mathbb{R}^{n}$ is the coordinate vector of point number $i$ in $\mathcal{X}$. Thus, there is a natural isomorphism between $\mathcal{C}_{m}^{n}$ and $\mathbb{R}^{m n}$. As topology on $\mathcal{C}_{m}^{n}$, we will use the one inherited from $\mathbb{R}^{m n}$.

Let $G$ be a group of transformations $\mathcal{C}_{m}^{n} \rightarrow \mathcal{C}_{m}^{n}$. By the $\mathbf{G}$-orbit of $\mathcal{X} \in \mathcal{C}_{m}^{n}$ is meant the set

$$
\{\mathcal{Y} \mid \mathcal{Y}=g(\mathcal{X}), g \in G\}
$$

We write $\mathcal{X} \sim \mathcal{Y}$, when $\mathcal{X}$ and $\mathcal{Y}$ are in the same orbit. Since $G$ is a group, this is an equivalence relation.

For a group $G$ of transformations $\mathbb{R}^{n} \rightarrow \mathbb{R}^{n}$, let $G^{m}$ be the group of product transformations, $\mathcal{C}_{m}^{n} \rightarrow \mathcal{C}_{m}^{n}$, defined by

$$
g\left(\mathcal{X}^{\prime}\right)=\left(g\left(p_{1}\right), \ldots, g\left(p_{m}\right)\right), \text { when } \mathcal{X}=\left(p_{1}, \ldots, p_{m}\right) \in \mathcal{C}_{m}^{n} \text { and } g \in G
$$

For our applications, this is the most usual situation, i.e. the same transformation is applied to all points of the configuration. By abuse of language, we write $G$ instead of $G^{m}$. 
For the geometric applications we are interested in, the following terminology is convenient.

Definition 1. Let $G$ be a group of transformations $\mathcal{C}_{m}^{n} \rightarrow \mathcal{C}_{m}^{n}$. The shape space is defined as the set of orbits $\mathcal{C}_{m}^{n} / G$. Let

$$
s: \mathcal{C}_{m}^{n} \rightarrow \mathcal{C}_{m}^{n} / G,
$$

be the natural projection. For $\mathcal{X} \in \mathcal{C}_{m}^{n}$, the orbit $s(\mathcal{X}) \in \mathcal{C}_{m}^{n} / G$ is called the shape of $\mathcal{X}$ and each element of $s(\mathcal{X})$ is called a representative of $s(\mathcal{X})$. A function $\varphi$ on $\mathcal{C}_{m}^{n}$, which is constant on each orbit is called an invariant. A set of functions $\varphi=\left(\varphi_{1}, \ldots, \varphi_{k}\right)$ is said to form a complete set of invariants if $\varphi(\mathcal{X})=\varphi(\mathcal{Y})$ if and only if $\mathcal{X} \sim \mathcal{Y}$. As topology on $\mathcal{C}_{m}^{n} / G$, we choose the strongest topology that makes s continuous.

Since $G$ forms a group, the orbits of $\mathcal{C}_{m}^{n} / G$ are disjoint. The space $\mathcal{C}_{m}^{n}$ can then be viewed as divided into disjoint strings, where each string is an orbit, see Figure 1.

Example 1. Let $G$ be the group of nonsingular Euclidean transformations, acting on $\mathcal{C}_{m}^{n}$. Euclidean invariants are distances between points in the point configurations. The orbits consist of configurations that are congruent, in the sense of Euclidean geometry.

\section{Density of shape, general theory}

We now introduce a parametrisation of point configurations $\mathcal{C}_{m}^{n}$, by taking the transformation group $G$ into account, which will enable us to write down a formula for a density function on the shape space $\mathcal{C}_{m}^{n} / G$, when a density function on $\mathcal{C}_{m}^{n}$, or on the individual points of the configurations is known. This parametrisation will be done by pulling $\mathcal{C}_{m}^{n}$ back to two parameter sets $A$ and $B$, such that $B$ labels the orbits $\mathcal{C}_{m}^{n} / G$ and $A$ provides parameters along each orbit. Recall that a function $f$ is called a $C^{n}$-diffeomorphism if it is invertible and both $f$ and $f^{-1}$ belong to $C^{n}$.

Definition 2. Let $G$ be a group of transformations $\mathcal{C}_{m}^{n} \rightarrow \mathcal{C}_{m}^{n}$. Suppose that there exist open sets $A \subseteq \mathbb{R}^{k}$ and $B \subseteq \mathbb{R}^{k^{\prime}}$ for some $k$ and $k^{\prime}$, a bijection

$$
g: A \ni a \rightarrow g_{a} \in G
$$

and a function

$$
\zeta: B \rightarrow \mathcal{C}_{m}^{n}
$$

such that the function

$$
\gamma_{\zeta}: A \times B \rightarrow \mathcal{C}_{m}^{n}, \text { defined by } \gamma_{\zeta}(a, b)=g_{a} \circ \zeta(b),
$$

has a dense open range $U \subseteq \mathcal{C}_{m}^{n}$ and $\gamma_{\zeta}: A \times B \rightarrow U$, is a $C^{1}$-diffeomorphism. Then we say that $\zeta$ a parametrisation of shape and $g$ a parametrisation of $G$. 
The dimensions $k, k^{l}$ only depend on $\mathcal{C}_{m}^{n}$ and $G$, as $\gamma_{\zeta}$ is a $C^{1}$-diffeomorphism. The specific choices of parametrisations for shape and $G$ merely represent a choice of coordinates on $U \subseteq \mathcal{C}_{m}^{n}$, such that $\zeta$ labels the orbits and $g$ describes the location within each orbit, see Figure 1.

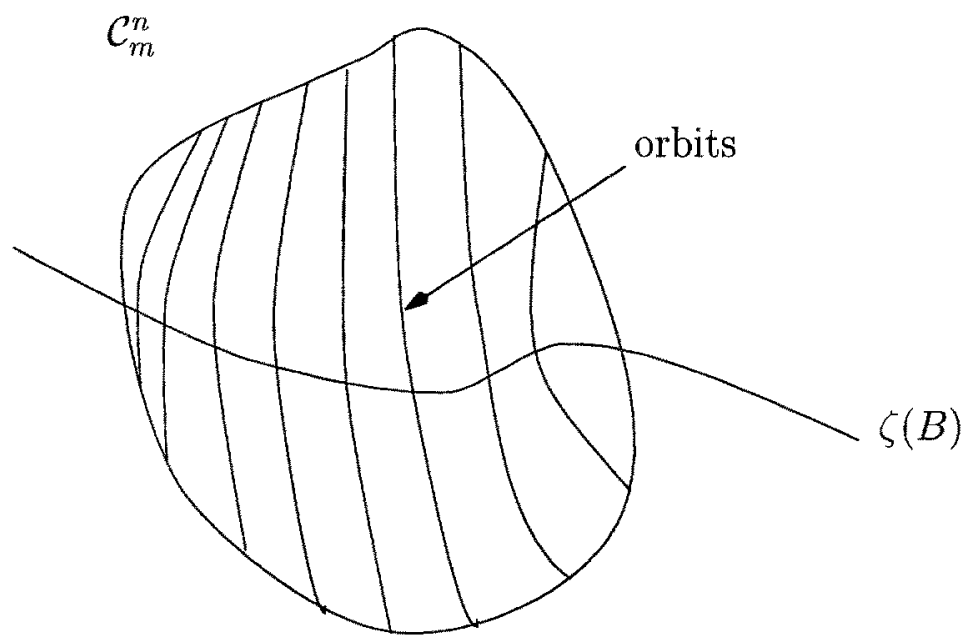

Fig. 1. Parametrisation of $\mathcal{C}_{m}^{n}$. Each orbit has a unique intersection with $\zeta(B)$.

We now turn to the main question, to define densities on shape spaces. To this end assume that $\mathcal{C}_{m}^{n}$ has a density function $0 \leq \phi \in L^{1}\left(\mathcal{C}_{m}^{n}\right)$, where $\int \phi d \mathcal{X}=$ 1. Often it is obtained from density functions for the individual points of the configurations. We want to define a density function on $\mathcal{C}_{m}^{n} / G$, i.e. a density function for shapes. Let $\gamma_{\zeta}$ be as in Definition 2, then

$$
\int_{\mathcal{C}_{m}^{n}} \phi(\mathcal{X}) d \mathcal{X}=\int_{B}\left(\int_{A} \phi \circ \gamma_{\zeta}(a, b)\left|\operatorname{det}\left(\gamma_{\zeta}^{\prime}\right)\right| d a\right) d b,
$$

where $\operatorname{det}\left(\gamma_{\zeta}^{\prime}\right)$ is the functional determinant of $\gamma_{\zeta}(a, b)$. It is thus a determinant of order $k+k^{\prime}$. Here the function

$$
B \ni b \rightarrow \int_{A} \phi \circ \gamma_{\zeta}(a, b)\left|\operatorname{det}\left(\gamma_{\zeta}^{\prime}\right)\right| d a \in L^{1}
$$

is defined almost everywhere. We use this function when defining a density of shape.

Definition 3. Let $\phi \in L^{1}\left(\mathcal{C}_{m}^{n}\right)$ be a density function, $\zeta: B \rightarrow \mathcal{C}_{m}^{n}$ a parametrisation of shape, $g: A \ni a \rightarrow g_{a} \in G$ a parametrisation of the group $G$ and set $\gamma_{\zeta}(a, b)=g_{a} \circ \zeta(b)$. The function $\mathfrak{D}(\phi, \zeta): B \rightarrow \mathbb{R}$, defined by

$$
\mathfrak{D}(\phi, \zeta): b \rightarrow \int_{A} \phi \circ \gamma_{\zeta}(a, b)\left|\operatorname{det} \gamma_{\zeta}^{\prime}\right| d a
$$


is called the density of shape on $\mathcal{C}_{m}^{n}$, with respect to $G$.

Observe that the density of shape depends on the parametrisation of shape, which can be chosen in many ways. This seems to bring in an ambiguity. Also the parametrisation of $G$ can be done in many ways. The following theorem clarifies the rate of ambiguity,

\section{Theorem 1.}

1. The density of shape is independent of the parametrisation of $G$.

2. If $\zeta_{i}: B_{i} \rightarrow \mathcal{C}_{n}^{n}, i=1,2$, are two parametrisations of shape, then there exist open and dense subsets $\tilde{B}_{i} \subseteq B_{i}$ and a $C^{1}$-diffeomorphism $f: \tilde{B}_{2} \rightarrow \tilde{B}_{1}$, such that

$$
\mathfrak{D}\left(\phi, \zeta_{2}\right)=\left|\operatorname{det} f^{\prime}\right| \mathfrak{D}\left(\phi, \zeta_{1}\right) \circ f \text {. }
$$

The second statement in Theorem 1 means that the dependence of $\zeta$ is illusory. In fact, the formula says that if we integrate over a set in $\mathcal{C}_{m}^{n} / G$ the result will be independent of the specific choice of parametrisation of shape $\zeta$.

Example 2. As an illustration of Definition 2 and 3 , let $G$ be the group of nonsingular affine transformations $\mathbb{R}^{2} \rightarrow \mathbb{R}^{2}$. Extend $G$ to the space $\mathcal{C}_{4}^{2}$, which consist of all ordered four point configurations in $\mathbb{R}^{2}$. Using matrix notation, we write

$$
\mathcal{X}=\left(\begin{array}{llll}
x_{1} & x_{2} & x_{3} & x_{4} \\
y_{1} & y_{2} & y_{3} & y_{4}
\end{array}\right) \in \mathcal{C}_{4}^{2}
$$

where each column represents a point in $\mathbb{R}^{2}$. Let

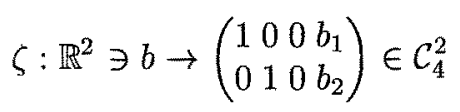

be a parametrisation of shape and

$$
g: A \ni a \rightarrow g_{a}(\cdot)=\left(\begin{array}{cc}
a_{11} & a_{12} \\
a_{21} & a_{22}
\end{array}\right)(\cdot)+\left(\begin{array}{c}
a_{1} \\
a_{2}
\end{array}\right) \in G, \quad \operatorname{det}\left(\left\{a_{i j}\right\}\right) \neq 0,
$$

be a parametrisation of $G$, where $A \subset \mathbb{R}^{6}$ is open and dense. The reason for choosing $\zeta$ as above is that almost every $\mathcal{X} \in \mathcal{C}_{4}^{2}$ can be transformed into the range of $\zeta$ by some $g_{a}$.

Now $\gamma_{\zeta}$ in Definition 2 is given by

$$
\gamma_{\zeta}: A \times \mathbb{R}^{2} \ni(a, b) \rightarrow\left(\begin{array}{lll}
a_{11} & a_{12} & a_{1} \\
a_{21} & a_{22} & a_{2}
\end{array}\right)\left(\begin{array}{cccc}
1 & 0 & 0 & b_{1} \\
0 & 1 & 0 & b_{2} \\
1 & 1 & 1 & 1
\end{array}\right) \in \mathcal{C}_{4}^{2} .
$$

$\gamma_{\zeta}$ has a dense open range in $\mathcal{C}_{4}^{2}$ and $\gamma_{\zeta}: A \times \mathbb{R}^{2} \rightarrow \gamma_{\zeta}\left(A \times \mathbb{R}^{2}\right)$ is a $C^{1}$. diffeomorphism.

If $\phi \in L^{1}\left(\mathcal{C}_{4}^{2}\right)$ is a density function, inserting $\gamma_{\zeta}$ in Definition 3 , gives us a density function of affine shapes for $\mathcal{C}_{4}^{2}$. 
The following theorem addresses the problem of finding the density of shape, when the density of $\mathcal{C}_{m}^{n}$ has been transformed by a change of coordinates.

Theorem 2. Let $\phi \in L^{1}\left(\mathcal{C}_{m}^{n}\right)$, be a density function, $g: A \rightarrow g_{a} \in G$ a parametrisation of $G$ and $\zeta$ a parametrisation of shape. If $g_{a_{0}} \in G$ is a $C^{1}$ diffeomorphism, then

$$
\mathfrak{D}(\phi, \zeta)=\mathfrak{D}\left(\left|\operatorname{det} g_{a_{0}}^{\prime}\right| \phi \circ g_{a_{0}}, \zeta\right) .
$$

As a consequence of Theorem 2, neither scaling nor translation of the density $\phi \in L^{1}\left(\mathcal{C}_{m}^{n}\right)$, affect the density of affine or positive similarity shape.

\section{Examples of densities of affine and similarity shape}

We will now focus on the affine and positive similarity groups of transformations.

Example 3 (Aff. shape, four points on a line, normally distributed). In this example, we compute the density of affine shape for four points on a line, when the points are normally and independently distributed. The density of shape will be a real valued function defined on $\mathbb{R}^{2}$.

Let

$$
\phi(x)=\frac{1}{\pi^{2}} \exp \left(-\sum_{i=1}^{4} x_{i}^{2}\right),
$$

be the density function on $\mathcal{C}_{4}^{1}$, in the case of four independent, normally distributed points on a line $l$, with equal means. Let $G$ be the group of nonsingular affine transformations $l \rightarrow l$. Furthermore, let $A=\left\{(a, t) \in \mathbb{R}^{2} \mid a \neq 0\right\}$. We parametrise $G$ by

$$
g: A \ni \alpha=(a, t) \rightarrow g_{\alpha}(\cdot)=a \cdot+t \in G,
$$

and as a parametrisation of shape we choose

$$
\zeta: \mathbb{R}^{2} \ni(x, y) \rightarrow(10 x y) \in \mathcal{C}_{4}^{1} .
$$

Set

$$
\gamma_{\zeta}: A \times \mathbb{R}^{2} \ni(\alpha, x, y) \rightarrow g_{\alpha} \circ \zeta(x) \in \mathcal{C}_{4}^{1} .
$$

Then the density of affine shape is

$$
\mathfrak{D}(\phi, \zeta)(x, y)=\int_{A} \phi \circ \gamma_{\zeta}(\alpha, x, y) a^{2} d \alpha
$$

which yields

$$
\mathfrak{D}(\phi, \zeta)(x, y)=\frac{1}{\pi^{2}} \int_{A} \exp \left\{(a+t)^{2}+t^{2}+(a x+t)^{2}+(a y+t)^{2}\right\} d a d t .
$$


A symbolic computation, e.g. by means of the computer program Maple, gives

$$
\mathfrak{D}(\phi, \zeta)(x, y)=\frac{2}{\pi}\left(3\left(1+x^{2}+y^{2}\right)-2(x+y+x y)\right)^{-3 / 2} .
$$

More generally, if the densities of the four points have different means $m_{i}$, $i=1,2,3,4$, but still equal standard deviations, i.e.

$$
\phi(x)=\frac{1}{\pi^{2}} \exp \left(-\sum_{i=1}^{4}\left(x_{i}-m_{i}\right)^{2}\right),
$$

then

$$
\mathfrak{D}(\phi, \zeta)(x, y)=\frac{2}{\pi} p^{-3 / 2} M\left(1+\frac{q}{2}\right)
$$

where

$$
\begin{gathered}
p=3\left(1+x^{2}+y^{2}\right)-2(x+y+x y), \\
q=\left\{-3 m_{1}+m_{2}+m_{3}+m_{4}+y\left(m_{1}+m_{2}+m_{3}-3 m_{4}\right)+\right. \\
\left.x\left(m_{1}+m_{2}-3 m_{3}+m_{4}\right)\right\}^{2} p^{-1}
\end{gathered}
$$

and

$$
M=\exp \left(\frac{1}{4}\left(\sum_{i=1}^{4} m_{i}\right)^{2}-\sum_{i=1}^{4} m_{i}^{2}+\frac{1}{4} q\right) .
$$

It is also possible to obtain densities of affine shape, in analytic form, for four points in a plane $\mathcal{C}_{4}^{2}$, when the points are independently normally distributed, or when the points are independently and identically uniformly distributed c.f. [2]. These densities can be used as a powerful tool for obtaining confidence sets for elements in a database, that contain shapes of feature points of objects.

Example 4 (Pos. sim. shape, three points in a plane, normally distributed). In this example, we compute the density of positive similarity shape for three points in plane, when the points are normally and independently distributed.

Let $G$ be the group of nonsingular positive similarity transformations $\mathbb{R}^{2} \rightarrow$ $\mathbb{R}^{2}$, with parametrisation

$$
g: A \ni \alpha=\left(a, b, t_{1}, t_{2}\right) \rightarrow g_{\alpha}(\cdot)=\left(\begin{array}{cc}
a & -b \\
b & a
\end{array}\right)(\cdot)+\left(\begin{array}{c}
t_{1} \\
t_{2}
\end{array}\right) \in G, \quad a^{2}+b^{2} \neq 0
$$

and

$$
\zeta: \mathbb{R}^{2} \ni(x, y) \rightarrow\left(\begin{array}{lll}
1 & 0 & 0 \\
0 & x & y
\end{array}\right) \in \mathcal{C}_{3}^{2},
$$

a parametrisation of shape. Set

$$
\gamma_{\zeta}: A \times \mathbb{R}^{2} \ni(\alpha, x, y) \rightarrow g_{\alpha} \circ \zeta(x, y) \in \mathcal{C}_{3}^{2} .
$$

The density of shape is

$$
\mathfrak{D}(\phi, \zeta)(x, y)=\int_{A}\left(a^{2}+b^{2}\right)|x-y| \phi \circ \gamma_{\zeta}(\alpha, x, y) d \alpha .
$$


Let

$$
\phi(x, y)=\frac{1}{\pi^{3}} \exp \left(-\sum_{i=1}^{3}\left(x_{i}^{2}+y_{i}^{2}\right)\right) \in L^{1}\left(\mathcal{C}_{3}^{2}\right)
$$

be the normal density function on $\mathcal{C}_{3}^{2}$, having equal mean and standard deviation. The density of shape is

$$
\mathfrak{D}(\phi, \zeta)(x, y)=\frac{3|x-y|}{4 \pi\left(1+x^{2}+y^{2}-x y\right)^{2}} .
$$

If we allow the points to have different means $\left(m_{x_{i}}, m_{y_{i}}\right), i=1,2,3$ but equal deviations, i.e.

$$
\phi(x, y)=\frac{1}{\pi^{3}} \exp \left(-\sum_{i=1}^{3}\left(\left(x_{i}-m_{x_{i}}\right)^{2}+\left(y_{i}-m_{y_{i}}\right)^{2}\right)\right) \in L^{1}\left(\mathcal{C}_{3}^{2}\right),
$$

then the density of shape becomes

$$
\mathfrak{D}(\phi, \zeta)(x, y)=\frac{3|x-y|}{4 \pi p^{2}}\left(1+\frac{1}{3} \frac{q_{1}^{2}+q_{2}^{2}}{2 p}\right) C_{1} C_{2} e^{2 r / 3},
$$

where

$$
\begin{gathered}
p=1+x^{2}+y^{2}-x y \\
q_{1}=2 m_{y_{1}}-m_{y_{2}}-m_{y_{3}}+\left(m_{x_{1}}+m_{x_{2}}-2 m_{x_{3}}\right) y+\left(m_{x_{1}}-2 m_{x_{2}}+m_{x_{3}}\right) x, \\
q_{2}=2 m_{x_{1}}-m_{x_{2}}-m_{x_{3}}+\left(2 m_{y_{2}}-m_{y_{1}}-m_{y_{3}}\right) x+\left(2 m_{y_{3}}-m_{y_{2}}-m_{y_{1}}\right) y \\
C_{i}=e^{q_{i}^{2} /(6 p)}, \quad i=1,2,
\end{gathered}
$$

and

$$
r=-\sum_{i=1}^{3}\left(m_{x_{i}}^{2}+m_{y_{i}}^{2}\right)+\sum_{\substack{i, j=1 \\ i<j}}^{3}\left(m_{x_{i}} m_{x_{j}}+m_{y_{i}} m_{y_{j}}\right)
$$

\section{Density of projective shape}

When $G$ is the group of nonsingular projective transformations $\mathbb{R}^{n} \rightarrow \mathbb{R}^{n}$, it is possible to obtain a density of projective shape for point configurations in $\mathcal{C}_{m}^{n}$, when $m \geq n+3$, c.f. [2]. We will, however, focus on projective invariants, for point configurations in $\mathcal{C}_{n+3}^{n}$, here. It can be shown, that there are $n$ such independent invariants. The following theorem gives the densities for these. For the special case of $\mathcal{C}_{4}^{1}$, the invariant will be the well known cross ratio.

Theorem 3. Let $\phi \in L^{1}\left(\mathcal{C}_{n+3}^{n}\right)$ be a density function and

$$
\zeta: \mathbb{R}^{n} \times \mathbb{R}^{n} \ni(x, y) \rightarrow(I 0 x y) \in \mathcal{C}_{n+3}^{n}
$$

a parametrisation of affine shape, where $I$ is the $n \times n$ identity matrix and 0 the $n \times 1$ matrix consisting of zeros. Then the density for projective invariants are given by: 
(i)

$$
\begin{aligned}
& \psi\left(\eta_{i}\right)=\int \mathfrak{D}(\phi, \zeta)\left(x, \eta_{i} t x_{1}, y_{2} \cdots, y_{i-1}, t x_{i}, y_{i+1}, \cdots, y_{n}\right)\left|t x_{1} x_{i}\right| d x d y d t \quad \text { a.e., } \\
& \text { for } i=2, \cdots, n .
\end{aligned}
$$

(ii)

$$
\begin{aligned}
& \psi\left(\eta_{n+1}\right)=\frac{1}{\left|\eta_{n+1}-1\right|} \int \mathfrak{D}(\phi, \zeta)\left(\alpha, x_{2} \cdots, x_{n}, t \eta_{n+1} \alpha, y_{2} \cdots, y_{n}\right)|\alpha \beta| d x d y d t \quad \text { a.e., } \\
& \text { where } x=\left(x_{2}, \cdots, x_{n}\right), y=\left(y_{2}, \cdots, y_{n}\right), \\
& \qquad \alpha=\frac{t\left(\sum_{2}^{n} x_{i}-1\right)-\left(\sum_{2}^{n} y_{i}-1\right)}{\left(\eta_{n+1}-1\right) t}
\end{aligned}
$$

and

$$
\beta=\frac{\left(\sum_{2}^{n} y_{i}-1\right)-\eta_{n+1} t\left(\sum_{2}^{n} x_{i}-1\right)}{\left(\eta_{n+1}-1\right) t} .
$$

Densities for the cross ratio have been computed before, for the situation when the points are normally and independently distributed with identical means and deviations, [6]. For the situation of uniform density for the points, see [1]. The appearances of these two densities are very similar and there are in fact many features for the cross ratio that generally apply when the points are distributed with the same densities. For example, the density of the cross ratio is symmetric with respect to $x=1 / 2$, which was pointed out in [6], and it is also easy to see that the density tends to infinity at $x=0$ and $x=1$. In fact, the following more general result shows when the density function tends to infinity at $x=0$ and $x=1$, even when the points are not identically distributed.

Theorem 4. Let $\Phi \in L^{1}\left(\mathcal{C}_{4}^{1}\right)$ be a density function for four points on a line, defined by

$$
\Phi(x)=\prod_{i=1}^{4} \phi_{i}\left(x_{i}\right),
$$

where $\phi_{i}$ are one dimensional densities. Let $M \in \mathbb{R}$ and let $\psi$ be the density for the cross ratio. Then

1. if

$$
\int \phi_{1}(x) \phi_{2}(x) d x>0, \int \phi_{3}(x) \phi_{4}(x) d x>0,
$$

there exists an open neighbourhood $U_{1} \ni 1$, such that $\psi(x)>M$ for almost all $x \in U_{1}$.

2. If

$$
\int \phi_{1}(x) \phi_{3}(x) d x>0, \int \phi_{2}(x) \phi_{4}(x) d x>0,
$$

then there exists an open neighbourhood $U_{0} \ni 0$, such that $\psi(x)>M$ for almost all $x \in U_{0}$. 
Example 5. For four points on a line, the cross ratio is a projective invariant. It can be shown that this is exactly the (ii) density in Theorem 3, with

$$
\alpha=\frac{(1-t)}{(k-1) t}
$$

and

$$
\beta=\frac{k t-1}{(k-1) t}
$$

Let $\phi \in L^{1}\left(\mathcal{C}_{4}^{\mathbf{1}}\right)$ be a density function, $\zeta$ a parametrisation of affine shape as in Theorem 3 and $\mathfrak{D}(\phi, \zeta)$ the corresponding affine density of shape. Then, the density of the cross ratio is

$$
\psi(k)=\frac{1}{|k-1|} \int \mathfrak{D}(\phi, \zeta)(\alpha, t k \alpha)|\alpha \beta| d t .
$$

We can for instance apply this to Example 3, where the density of affine shape for four normally and independently distributed points, with different means, is given.

\section{References}

1. K. Åström. Invariancy Methods for Points, Curves and Surfaces in Computer Vision. PhD thesis, Lund University, Lund Institute of Technology, Department of Mathematics, 1996.

2. R. Berthilsson. A statistical theory of shape. Technical report, Department of Mathematics, Lund Institute of Technology, 1997. Licentiate Thesis, www.maths.lth.se/matematiklth/personal/rikard/index.html.

3. Anders Heyden. Geometry and Algebra of Multiple Projective Transforms. PhD thesis, Lund University, Lund Institute of Technology, Department of Mathematics, 1995.

4. David G. Kendall. A survey of the statistical theory of shape. Statistical Science, $4(2): 87-120,1989$.

5. V. Mardia, K. and I. L. Dryden. Shape distribution for landmark data. Adv. Appl. Prob., pages 742-755, 1989.

6. S. J. Maybank. Probabilistic analysis of the application of the cross ratio to model based vision: Misclassification. International Journal of Computer Vision, 14(3), 1995.

7. Carl-Gustav Werner. Selective geometric hashing, by means of determinants of transformations. In SCTA '93, Proceedings of the 8th Scandinavian Conference on Image Analysis, volume 2, pages 715-718. NOBIM, 1993. 